\title{
Development of a Probe Based on Quantum Dots Embedded with Molecularly Imprinted Polymers to Detect Parathion
}

\author{
Jianshe Tang ${ }^{1,2 *}$, Li Xiang1 \\ ${ }^{1}$ Department of Environment and Energy Engineering, Anhui Jianzhu University, \\ Hefei 230601, The People's Republic of China \\ ${ }^{2}$ Key Laboratory of Water Pollution Control and Waste Water Resources in Anhui Province, \\ Hefei 230601, The People's Republic of China
}

Received: 22 October 2015

Accepted: 5 December 2015

\begin{abstract}
Surface molecularly imprinted CdTe nanoparticles with molecular recognitive activity were prepared by reverse microemulsion polymerization with parathion as template molecules, 3-aminopropyltriethoxysilane and tetramethoxysilane as the polymerization precursors, and cross-linking. The determination method for parathion was developed on the basis of the fluorescence quenching of quantum dots caused by parathion due to the binding of molecularly imprinted cavities to parathion. The synthesized material had a distinguished selectivity and high binding affinity to parathion compared with chlopyrifos, diazinon, and pyrimithate. Under optimal conditions, the relative fluorescence intensity of polymers decreased with increases of the concentration of parathion in the range $0.05-1000 \mu \mathrm{mol} / \mathrm{L}$. The decreasing tendency of fluorescence intensity with increasing parathion concentration abides by the logistical growth curve with a detected limit of $0.218 \mu \mathrm{mol} / \mathrm{L}$. This method was used to detect for parathion in water samples, for which recoveries ranging from $97.72 \%$ to $100.59 \%$ were obtained.
\end{abstract}

Keywords: fluorescence quenching, molecularly imprinted polymers, sensors, determination, quantum dots

\section{Introduction}

Organophosphorous pesticides (OPs) are a broad group of chemicals widely used in agriculture. OPs irreversibly inhibit acetylcholinesterase (AchE), which is essential to the nerve function in insects, humans, and many other animals [1]. Parathion, as one of the most

*e-mail: tjs28@ahjzu.edu.cn important OPs, consists of a thiophosphoric moiety linked to a nitrobenzene group. Its toxicity is primarily associated with inhibition of AchE and leads to excessive cholinergic neurotransmission and subsequent signs of toxicity, including autonomic dysfunction, involuntary movements, and, ultimately, death [2]. For human health protection and environmental control, it is an important challenge worldwide to develop a rapid, selective, and sensitive method for determining parathion.

Among the various detection techniques, fluorescent probes make the best choice, since they are qualified 
with high sensitivity, high selectivity, fast response, and with direct detection [3]. The combination of the specific recognition properties of molecularly imprinted polymers (MIPs) and the excellent optical properties of quantum dots (QDs) for recognition signal amplification and optical readout have drawn great attention. Coreshell magnetic fluorescent MIPs for the detection of traces of $\lambda$-cyhalothrin were prepared by copolymerization of acrylamide with a small quantity of allyl fluorescein in the presence of $\lambda$-cyhalothrin to form recognition sites without doping [4]. A similar method was developed for cyphenothrin [5]. In addition, a similar method for $\lambda$-Cyhalothrin on the basis of the fluorescence quenching of $\mathrm{YVO}_{4}: \mathrm{Eu}_{3}^{+} @ \mathrm{MIPs}$ has been developed, allowing us to achieve a determination limit as low as $1.76 \mu \mathrm{mol} / \mathrm{L}[6]$. The fluorescent CdTe/MIPs to the Bovine Hemoglobin were fabricated by 3-aminopropyltriethoxysilane (APTES) and tetramethoxysilane (TEOS) as the functional monomers and cross linker, respectively [7]. A recent review of QDbased sensors stated its usage based on chemosensors for ions and small molecules, and for biosensors as described by Cui [8]. Not only QDs, but two-photon fluorescent chemical molecular probes for $\beta$-galactosidase, plus a supramolecular glycoprobe for quick serological detection of a cancer biomarker also were prepared and have been reported previously $[9,10]$.

Although the MIPs to parathion were developed for an analogous enzyme-linked immunosorbent assay [11], the fluorescent probes for the determination of parathion based on couple grafting of functional MIPs on the surface of QDs has not yet been investigated. The purpose of this work was to develop a high affinity QDs@MIPs sensor for selective determination of trace parathion in water samples. We used CdTe QDs as supports, and used APTES, TEOS, and parathion as the functional monomer, crosslinker, and template, respectively, to produce surface graft imprinting materials. The developed QDs@MIPs could selectively bind parathion, which quickly quenches QD fluorescence, and therefore is capable of accurate quantification in a broad detection range. Moreover, this sensor had advantages of being more economical and eco-friendly as compared to chromatographic methods, and being faster and easier to use than immunoassay. The developed QDs@MIPs could provide a new and general strategy for the sensitive and selective detection of OPs.

\section{Experimental}

\section{Materials and Apparatus}

Unless otherwise stated, all chemicals used in this work were of analytical-reagent grade and used without further purification. Cadmium acetate dihydrate, sodium borohydride, sodium hydroxide, ammonium hydroxide, chloroform, cyclohexane, acetone, and ethanol were provided by Sinopharm Chemical Reagent Shanghai Co., Ltd. (Shanghai, China). Tellurium powder (99.99\%), APTES, TEOS, and thioglycollic acid (TGA) were purchased from Sigma-Aldrich Chemical Company (St. Louis, MO, USA). Parathion, chlopyrifos, diazinon, and pyrimithate were purchased from the Institute of Environmental Protection, Ministry of Agriculture (Tianjin, China). The structures of pesticides are shown in Fig. 1. Ultrapure water (18.3 M $\Omega$ ) obtained from Millipore Milli-Q purification system (Boston, America) was used to prepare solutions.

Fluorescence spectra were obtained at room temperature using an F7000 spectrofluorimeter (Hitachi, Japan) equipped with $1 \mathrm{~cm}$ quartz cuvette at slit width of $5 \mathrm{~nm}$. Absorption spectra were collected using a UV1800 PC UV-vis spectrophotometer (Jinhua, Shanghai) at room temperature. Morphological evaluation was examined with a transmission electron microscope (TEM, JEM2100F, Japan).

\section{Synthesis of CdTe QDs}

Thioglycollic acid-capped CdTe quantum dots were synthesized according to the reported method from the literature $[12,13]$. Briefly, $160 \mathrm{mg}$ of cadmium acetate dihydrate, $130 \mu \mathrm{L}$ of pure TGA, and $75 \mathrm{~mL}$ of distilled water were mixed in a three-necked flask to form the cadmium precursor. The addition of $1 \mathrm{~N} \mathrm{NaOH}$ drops was then used to adjust the $\mathrm{pH}$ to 12 under vigorous stirring for $30 \mathrm{~min}$. The cadmium and thiol solution was placed in a $150 \mathrm{~mL}$ three-neck flask with a thermometer and a large stirbar. Deaeration of the solution was performed under a robust flow of nitrogen with stirring at room temperature. A fresh aqueous solution of $\mathrm{NaHTe}$ was prepared by reacting $80 \mathrm{mg}$ of $\mathrm{NaBH}_{4}$ and $80 \mathrm{mg}$ of tellurium powder. Then 1 $\mathrm{mL} \mathrm{NaHTe}$ aqueous solution was injected into the reaction system under stirring. At that time the solution changed from clear to a bright, optically transparent orange tone.

\section{Preparation of MIP-Capped CdTe QDs (QDs@MIPs)}

Parathion-imprinted silica nanosphere embedded $\mathrm{CdTe}$ quantum dots were synthesized by modified reverse microemulsion methods [14]. $1.2 \mathrm{~mL}$ of Triton X-100

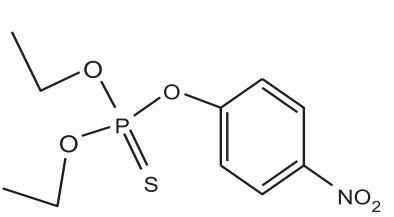<smiles>CCOP(=S)(OCC)Oc1nc(Cl)c(Cl)cc1Cl</smiles>

Parathion<smiles>CCOP(=S)(OCC)Oc1cc(C)nc(C(C)C)n1</smiles>

Clorpyrifos

Diazinon Pirimiphos ethyl

Fig. 1. The structure of pesticides. 
and $1.8 \mathrm{~mL}$ of chloroform were dispersed in $7.5 \mathrm{~mL}$ of cyclohexane, $500 \mu \mathrm{L}$ of CdTe QDs, and $60 \mu \mathrm{L}$ of ammonia to form a microemulsion solution. Next, $100 \mu \mathrm{L}$ of TEOS was added to initiate hydrolysis. After the mixture was stirred for $30 \mathrm{~min}, 1 \mathrm{~mL}$ of ethanol solution of $5 \mathrm{mg}$ of parathion and $25 \mu \mathrm{L}$ of APTES were added and the mixture was stirred for $24 \mathrm{~h}$. Finally, the microemulsion was broken by $10 \mathrm{~mL}$ of acetone, and the resultant precipitate was washed in sequence with ethanol and water. After removal of the supernatant, $3 \mathrm{~mL}$ of water was added, and the silica particles were regimented again by centrifuge for $20 \mathrm{~min}$. The nonimprinted polymer (NIP) was synthesized in parallel but without the addition of parathion.

\section{Analysis of Water Samples}

2.5 mL of QDs@MIPs and 0.5 mL of ultrapure water were added to the quartz cuvette. The concentration of QDs@MIPs was adjusted so that a fluorescence intensity of approximately 7,000 a.u. was achieved. To evaluate the QDs@MIPs binding capability to pesticides, $2.5 \mathrm{~mL}$ of QDs solution at uniform concentration and $0.5 \mathrm{~mL}$ of pesticides solution at different concentrations were mixed in a centrifuge tube and the fluorescence intensity was measured. The parallel experiments were performed with NIPs.

Surface river water samples were collected from local rivers. The samples were filtered through $0.45 \mu \mathrm{m}$ filters and stored in pre-cleaned glass bottles. As no parathion in the collected water samples was detectable by the proposed method, a recovery study was carried out on the samples spiked with 3-60 $\mu \mathrm{mol} / \mathrm{L}$ parathion to evaluate the developed method.

\section{Results and Discussion}

\section{Preparation of QD-kernelled MIPs}

Of polymeric materials, silica with a highly crosslinked rigid matrix and hydrophilic surface has a wide

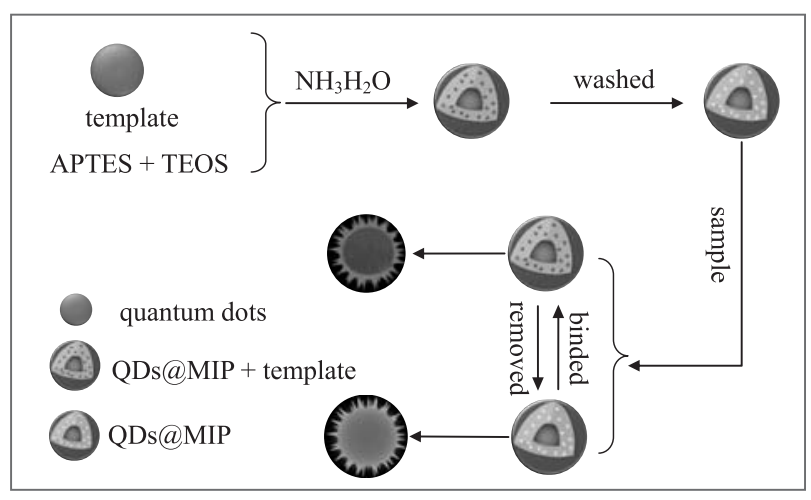

Fig. 2. Scheme of preparation and usage of QDs@MIPs. The upper part of the figure indicates that the preparation of QDs@ MIPs, and the lower part appeared the template quenching the fluorescence of QDs@MIPs. choice of functional precursors and structural forms $[14,15]$. In this work, we fabricated an imprinted silica matrix embedded with CdTe QDs, which could be adopted to monitor the target molecules by the changes of fluorescence intensity of CdTe QDs. MIP-capped CdTe QDs were prepared via a surface molecular imprinting process similar to a previously reported procedure [16-18]. The O, O-diethyl, and nitrophenyl groups of parathion can form strong hydrogen bonding with the amino groups of the functional monomer to form MIP film on the surface of QDs (Fig. 2).

The imprinting process would allow the formation of appropriately sized recognition cavities for parathion. The thickness of the MIP film is a key factor that affects site accessibility and mass-transfer resistance of an analyze [19]. The amount of TEOS used was found to be crucial for the sensitivity of parathion detection. A high amount of TEOS results in a large share of silica not dotted with QD, and thus in a correspondingly low intensity of the MIP fluorescence. On the other hand a too small amount gives insufficient capping of the template molecules and thus reduces the binding capacity of the QD@MIPs formed [20]. The TEOS:APTES $=3: 1$ ratio is optimized in our experiments.

The UV-vis absorption spectrum of QD@MIPs is shown in Fig. 3 and is identical to the absorption spectrum of free CdTe QD reported in the literature [21]. Fig. 3 also shows the fluorescence spectrum obtained upon excitation at $365 \mathrm{~nm}$ exhibiting a maximum at $556 \mathrm{~nm}$. The fluorescence property of the bared QD was unchanged by its incorporation into the MIP structure. This phenomenon was also discovered in the literatures of $[12,13]$. TEM images were taken for QDs@MIPs (Fig. 4). TEM images illustrated that the polymers are highly spherical and monodispersed with an average size of about $10 \mathrm{~nm}$.

\section{Fluorescence Quenching Analysis}

The prepared MIPs exhibited good fluorescence signal. Typically, MIPs had symmetric fluorescence emission at $556 \mathrm{~nm}$ obtained upon excitation at $365 \mathrm{~nm}$. As a recognition element, MIPs also exhibited the fluorescence quenching response to the template of parathion. The degree of fluorescence quenching is related to parathion concentration (Fig. 5).

The fluorescence quenching of MIP-QDs with various concentrations of parathion ranging from 0.05 to 1,000 $\mu \mathrm{mol} / \mathrm{L}$ was measured. We found that the fluorescence of the MIP-QDs gradually quenched with increasing concentrations of parathion. There was no overlap between the absorption band of parathion ( $\lambda \max 280 \mathrm{~nm} \mathrm{[22])}$ and the fluorescence emission band of the QDs, which was suited at $556 \mathrm{~nm}$, and so the fluorescence quenching mechanism of QDs induced by parathion was not fluorescence resonance energy transfer, [23] but probably electron transfer fluorescence quenching [18, 24]. In order to investigate the binding performance of the MIPs and NIPs based on hydrophobic CdTe QD nanomaterials, an equilibrium binding analysis was 

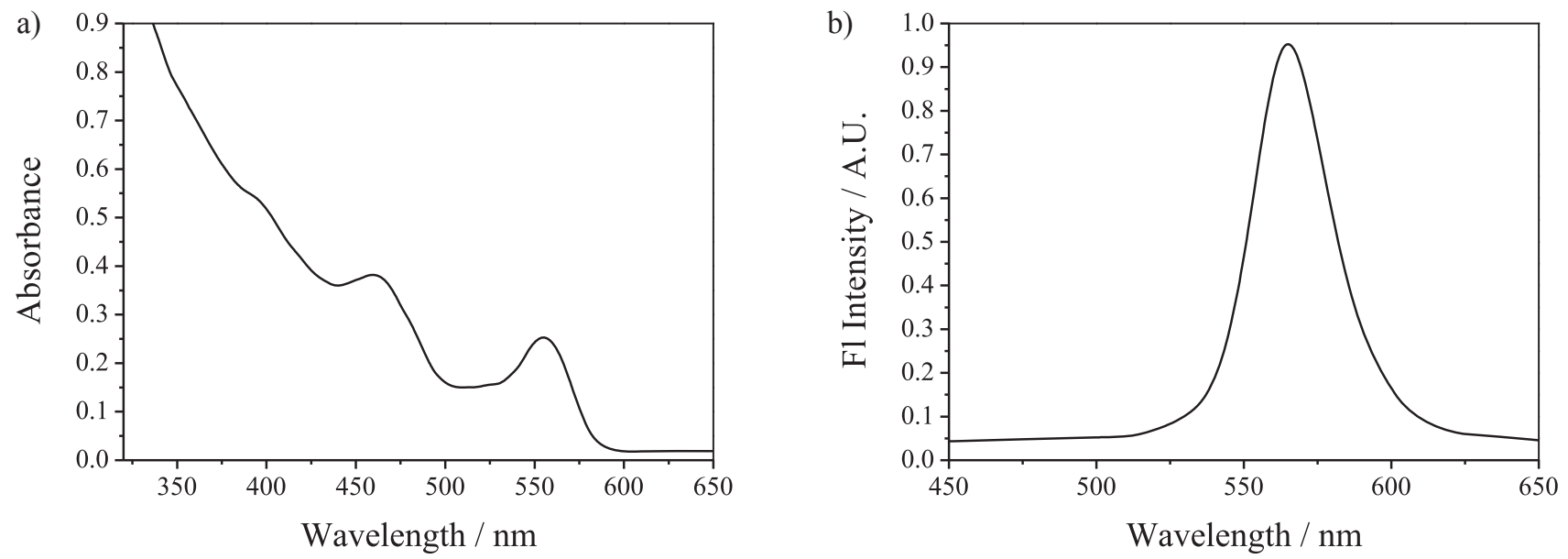

Fig. 3. (a) The absorbance spectrum and (b) fluorescence spectrum of QDs@MIPs.

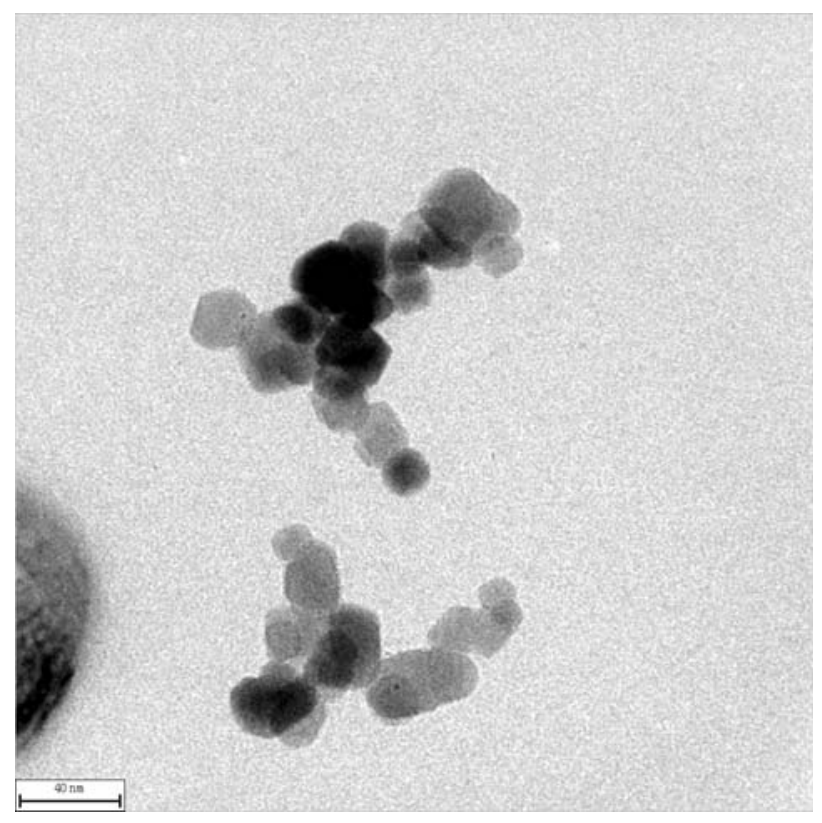

Fig. 4. TEM of QDs@MIPs.

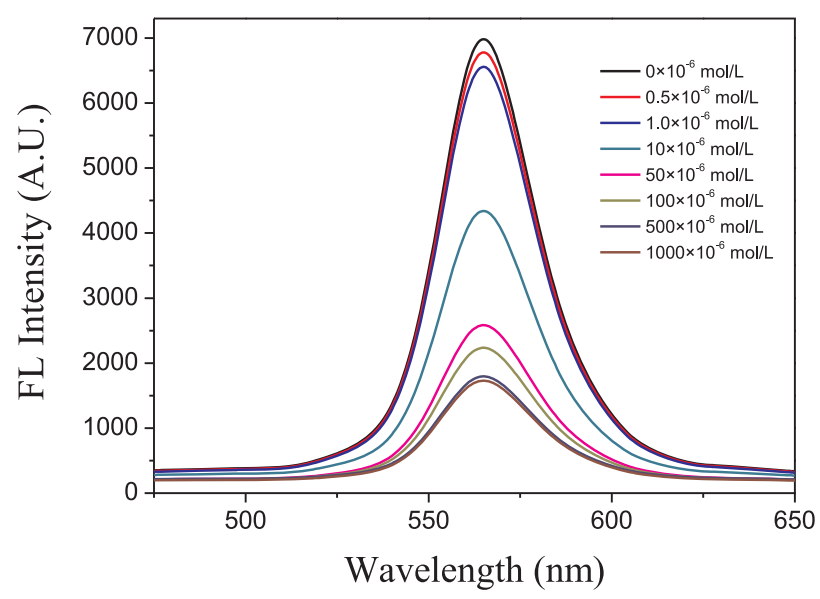

Fig. 5. Fluorescence spectra of MIP based on QDs@MIPs with increasing concentrations of parathion.
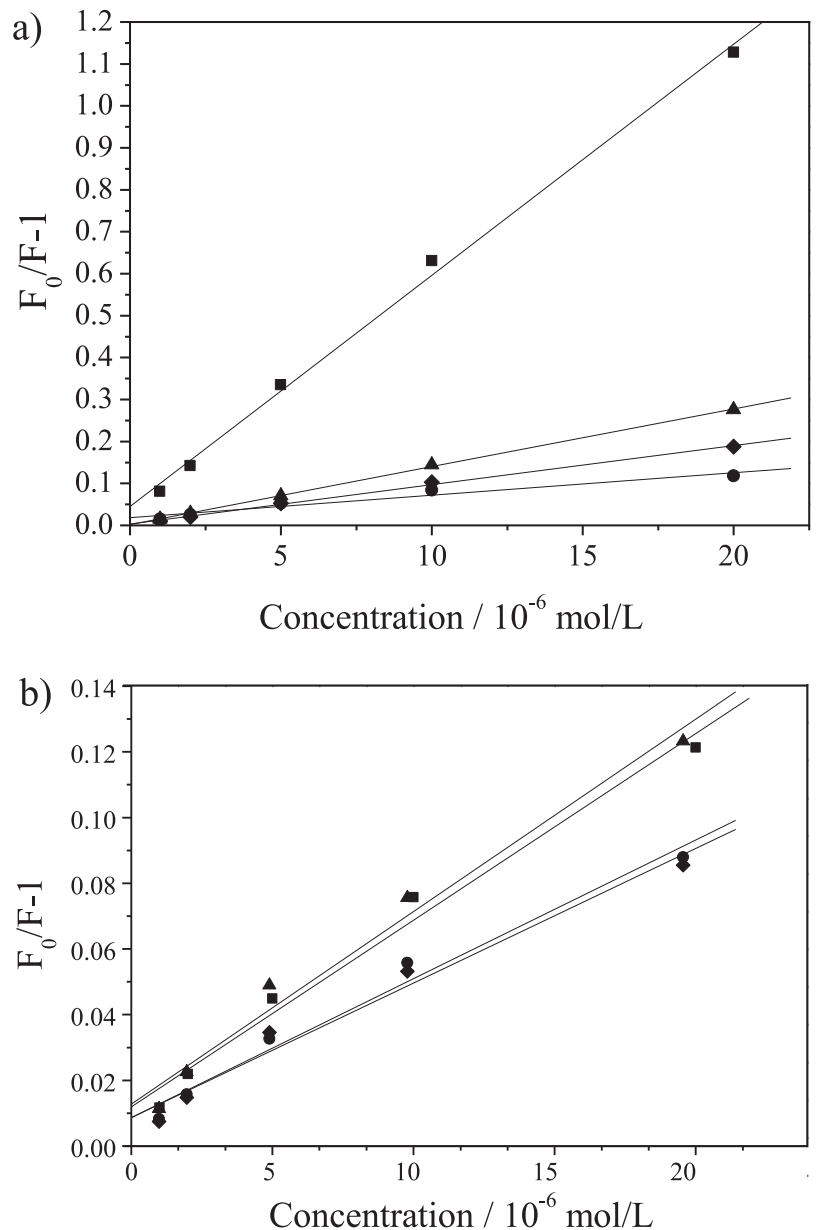

Fig. 6 Selectivity of (a) MIP and (b) NIP based on QDs@MIPs for parathion (square), chlopyrifos (triangle), diazinon (spheric), and pyrimithate (diamond). 


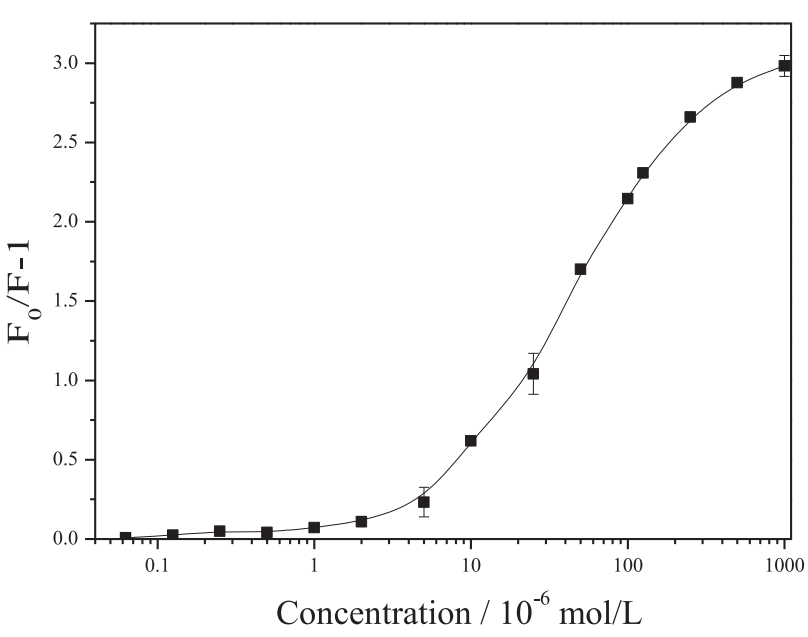

Fig. 7. Standard curve for parathion.

carried out using 1.0 20 $\mu \mathrm{mol} / \mathrm{L}$ of parathion, chlopyrifos, diazinon, and pyrimithate (Fig. 6). The analyte quenches the fluorescence of the QD in a concentration dependence in agreement with the Stern-Volmer equation as described in the literature $[14,16,25]$. The Stern-Volmer formula is given as:

$$
F_{0} / F=1+K s v[Q]
$$

...where $\mathrm{F}$ and $\mathrm{F}_{0}$ are the fluorescent intensities of the QDs@ MIPs at a given related analyte concentration and in an analyte-free solution, respectively. Ksv is the Stern-Volmer quenching constant, and $[Q]$ is the analyte concentration.

Fig. 7 illustrated the corresponding plot that showed a linear relationship between fluorescence response and parathion concentration in the range of 1.0 20 $\mu \mathrm{mol} / \mathrm{L}$ with a correlation coefficient of 0.9999 . The linear Stern-Volmer relationships of both MIPs and NIPs to four organphosphorus pesticides were observed with correlation coefficient range of 0.953-0.999. The Ksv of parathion in the case of QD@MIP is much higher than those of the other selected pesticides due to imprinted silica particles exhibiting the high specific binding ability for parathion compared to non-imprinted silica particles. This phenomenon showed evidence for an efficient imprinting effect of QDs@MIPs.
It is well known that the imprinting factor (IF) is an important index to evaluate the extent of quenching and sensitivity of the imprinted materials. IF is generally defined as $\alpha=K_{M T P_{s}} / K_{N I P S}$. Where $K_{M T P S}$ and $K_{N I P s}$ are adsorption capacities of the template on MIPs and NIPs, respectively [24]. The IF values were 8.73, 1.45, 1.35, and 1.43 for parathion chlopyrifos, diazinon, and pyrimithate, respectively (Table 1). High IF indicated that the cavities with binding sites in MIPs provided a better space for parathion. In addition, all of the analogues display little response to both MIPs and NIPs. The Ksv of analogues in MIPs was higher than that in NIPs. In view of these results, chlorpyrifos most likely competes strongest with parathion for the MIP binding sites.

\section{Determination of Parathion}

The primary researchers have pointed out that the standard curves based on fluorescence quenching could be done in terms of Stern-Volmer formulation $[6,26]$. The SternVolmer with good linear relationships fit under the narrow range of concentration. The four parameter logistic nonlinear regression model is commonly used for curvefitting analysis in bioassays [27-29]. It has been described as follows:

$$
F(x)=\frac{A-D}{1+(x / C)^{B}}+D
$$

...where $\mathrm{F}(\mathrm{x})$ is the response value (calculated as $\left.F_{0} / F-1\right), \mathrm{A}$ is the max response value in the present of highest concentration parathion, $\mathrm{D}$ is the least response value without parathion, $\mathrm{B}$ is slope, and $\mathrm{C}$ is parathion concentration corresponding half response effect.

In the concentration range of $0.05 \sim 1,000 \mu \mathrm{mol} / \mathrm{L}$ of parathion, the non-linear S-shape relationship between $F_{0} / F-1$ and parathion concentration was observed with

the regression equation of $F(x)=\frac{3.094-0.011}{1+(x / 44.624)^{1.051}}+0.011$

and a correlation coefficient of 0.9991 . The limit of detection (LOD), calculated as parathion concentration

Table 1. Fluorescence quenching constants of (a) MIP and (b) NIP based on QDs@MIPs for parathion, chlopyrifos, diazinon, and pyrimithate.

\begin{tabular}{|c|c|c|c|c|}
\hline Quenching constants & Parathion & Chlopyrifos & Diazinon & Pyrimithate \\
\hline$K s v_{\text {-MIP }}$ & 0.05839 & 0.01396 & 0.00654 & 0.00674 \\
\hline$K s v_{- \text {IIP }}^{*}$ & 0.00669 & 0.00962 & 0.00484 & 0.00472 \\
\hline $\mathrm{IF}^{* *}$ & 8.72795 & 1.45114 & 1.35124 & 1.42796 \\
\hline $\mathrm{K}^{* *}$ & 1 & 4.18266 & 8.92813 & 8.66320 \\
\hline
\end{tabular}

*: IF stands for the value of $K s v_{-\mathrm{MIP}} / K s v_{\text {-NIP }}$ in the same one pesticide.

**: K stands for the value of $K s v_{-\mathrm{MIP}}$ of parathion divided by $K s v_{\text {-MIP }}$ of chlopyrifos, diazinon, and pyrimithate, respectively. 
Table 2. Spiked recovery results for the determination of parathion in water samples $(n=7)$.

\begin{tabular}{|c|c|c|c|c|}
\hline \multirow{2}{*}{ Sample } & $\begin{array}{c}\text { Spiked } \\
\left(10^{-6} \mathrm{~mol} / \mathrm{L}\right)\end{array}$ & $\begin{array}{c}\text { Found } \\
\left(10^{-6} \mathrm{~mol} / \mathrm{L}\right)\end{array}$ & Recovery (\%) & RSD (\%) \\
\hline \multirow{3}{*}{ River water } & 0.5 & 0.49 & 97.72 & 2.6 \\
\cline { 2 - 5 } & 5 & 5.09 & 101.6 & 2 \\
\cline { 2 - 5 } & 10 & 9.9 & 100.59 & 2 \\
\cline { 2 - 5 } Tap water & 0.5 & 0.5 & 99.7 & 2.21 \\
\cline { 2 - 5 } & 5 & 4.96 & 99.29 & 2 \\
\hline
\end{tabular}

which produced enhancement three times the standard deviation of the blank signal, was $0.218 \mu \mathrm{mol} / \mathrm{L}$ and the limit of quantity (10 times the standard deviation of the blank signal) was $0.642 \mu \mathrm{mol} / \mathrm{L}$.

\section{Application to Real Water Sample Analysis}

In order to evaluate the feasibility of the proposed method in real sample detection, the QDs@MIPs sensor has been applied for the determination of parathion in tap water and river water samples. The results obtained by standard addition method were summarized in Table 2. It can be seen that the recoveries in the real water samples were between 97.72 and $100.59 \%$, and the relative standard deviation (RSD) was lower than $2.21 \%$. The above results demonstrated the good accuracy and precision of the developed method, which suggested that this sensor has the capability for detecting parathion in real water samples.

\section{Conclusion}

We have developed a novel quantum dots embedded MIPs for parathion to construct the fluorescence quenching MIPs-based probe to detect the parathion in water samples. The rebinding of the target analytes to the recognition sites of MIPs cavities thus resulted in the decreasing fluorescence emission of CdTe quantum dots. The QDs@MIPs integrated the high selectivity of MIPs and the strong fluorescence property of QDs. The simple, rapid, and reliable detecting method based on QDs@MIPs developed by QDs@MIPs opens up attractive perspectives for OPs monitoring.

\section{Acknowledgements}

The authors acknowledged the financial support by the National Natural Science Foundation of China (No. 21205001), Natural Science Foundation of Anhui Province (No. 1208085MB16), Key Technologies Research and Development Program of China (No. 2012BAJ08B03), and Competitive Project from Huizhou Construction Engineering Technology Research Center (No. 2012hjy04).

\section{References}

1. LIANG H., SONG D., GONG J., Signal-on electrochemiluminescence of biofunctional CdTe quantum dots for biosensing of organophosphate pesticides, Biosensors \& Bioelectronics, 53, 363, 2014.

2. CHUNYA L., CHANGFA W., YONG M., WEI B., SHENGSHUI H., A novel amperometric sensor and chromatographic detector for determination of parathion, Analytical and bioanalytical chemistry, 381, 1049, 2005.

3. HEMMILA I., LAITALA V., Progress in lanthanides as luminescent probes, Journal of Fluorescence, 15, 529, 2005.

4. GAO L., WANG J., LI X., YAN Y., LI C., PAN J., A coreshell surface magnetic molecularly imprinted polymers with fluorescence for lambda-cyhalothrin selective recognition, Analytical and Bioanalytical Chemistry, 406, 7213, 2014.

5. REN X., CHEN L., Quantum dots coated with molecularly imprinted polymer as fluorescence probe for detection of cyphenothrin, Biosensors \& Bioelectronics, 64, 182, 2015.

6. LIU C., SONG Z., PAN J., YAN Y., CAO Z., WEI X., GAO L., WANG J., DAI J., MENG M., YU P., A simple and sensitive surface molecularly imprinted polymers based fluorescence sensor for detection of lambda-Cyhalothrin, Talanta, 125, 14, 2014.

7. LI D.-Y., HE X.-W., CHEN Y., LI W.-Y., ZHANG Y.-K. Novel Hybrid Structure Silica/CdTe/Molecularly Imprinted Polymer: Synthesis, Specific Recognition, and Quantitative Fluorescence Detection of Bovine Hemoglobin, Acs Applied Materials \& Interfaces, 5, 12609, 2013.

8. CUI L., HE X.P., CHEN G.R. Recent progress in quantum d ot based sensors. Rsc Advances, 5, 26644, 2015.

9. LEE H.W., HEO C.H., SEN D., BYUN H.-O., KWAK I.H., YOON G., KIM H.M. Ratiometric two-photon fluorescent probe for quantitative detection of $\beta$-galactosidase activity in senescent cells. Analytical Chemistry, 86, 10001, 2014.

10. HE X.P., HU X.L., JIN H.Y., GAN J.M., ZHU H.L., LI J., LONG Y.T., TIAN H., Quick Serological Detection of a Cancer Biomarker with an Agglutinated Supramolecular Glycoprobe. Analytical Chemistry, 87, 9078, 2015.

11. TANG J.S., XIANG L., Development of a competitive format sorbent assay for the determination of parathion in water using molecular imprinted polymer as specific sorbent carrier, Chinese Chemical Letters, 21, 1361, 2010.

12. WANG C., MA Q., DOU W.C., KANWAL S., WANG G.N., YUAN P.F., SU X.G. Synthesis of aqueous CdTe quantum dots embedded silica nanoparticles and their applications as fluorescence probes, Talanta, 77, 1358, 2009.

13. XU S.F., LU H.Z., LI J.H., SONG X.L., WANG A.X., CHEN L.X., HAN S.B. Dummy Molecularly Imprinted PolymersCapped CdTe Quantum Dots for the Fluorescent Sensing of 
2,4,6-Trinitrotoluene, Acs Applied Materials \& Interfaces, 5, 8146, 2013.

14. LI H.B., LI Y.L., CHENG J. Molecularly Imprinted Silica Nanospheres Embedded CdSe Quantum Dots for Highly Selective and Sensitive Optosensing of Pyrethroids, Chemistry of Materials, 22, 2451, 2010.

15. XIE C.G., LIU B.H., WANG Z.Y., GAO D.M., GUAN G.J., ZHANG Z.P. Molecular imprinting at walls of silica nanotubes for TNT recognition, Analytical Chemistry, 80, 437, 2008.

16. WEI F.D., WU Y.Z., XU G.H., GAO Y.K., YANG J., LIU L.P., ZHOU P., HU Q. Molecularly imprinted polymer based on CdTe@SiO2 quantum dots as a fluorescent sensor for the recognition of norepinephrine, Analyst, 139, 5785, 2014.

17. YANG M., HAN A.J., DUAN J.L., LI Z.P., LAI Y.C., ZHAN J.H. Magnetic nanoparticles and quantum dots coloaded imprinted matrix for pentachlorophenol, Journal of Hazardous Materials, 237, 63, 2012.

18. CHEN Y.P., WANG D.N., YIN Y.M., WANG L.Y., WANG X.F., XIE M.X. Quantum Dots Capped with Dummy Molecularly Imprinted Film as Luminescent Sensor for the Determination of Tetrabromobisphenol A in Water and Soils, Journal of Agricultural and Food Chemistry, 60, 10472, 2012.

19. KARIM K., BRETON F., ROUILLON R., PILETSKA E.V., GUERREIRO A., CHIANELLA I., PILETSKY S.A. How to find effective functional monomers for effective molecularly imprinted polymers?, Advanced Drug Delivery Reviews, 57, 1795, 2005.

20. DAN L., WANG H.F. Mn-Doped ZnS Quantum Dot Imbedded Two-Fragment Imprinting Silica for Enhanced Room Temperature Phosphorescence Probing of Domoic Acid, Analytical Chemistry, 85, 4844, 2013.

21. WOLCOTT A., GERION D., VISCONTE M., SUN J., SCHWARTZBERG A., CHEN S.W., ZHANG J.Z. Silicacoated $\mathrm{CdTe}$ quantum dots functionalized with thiols for bioconjugation to IgG proteins, Journal of Physical Chemistry B, 110, 5779, 2006.

22. HIRT R.C., GISCLARD J.B. Determination of Parathion in Air Samples by Ultraviolet Absorption Spectroscopy, Analytical Chemistry, 23, 185, 1951.

23. LAKOWICZ J.R., Principles of Fluorescence Spectroscopy, third ed., springer, 2011.

24. LIU H.L., FANG G.Z., WANG S., Molecularly imprinted optosensing material based on hydrophobic CdSe quantum dots via a reverse microemulsion for specific recognition of ractopamine, Biosensors \& Bioelectronics, 55, 127, 2014.

25. WANG H.F., HE Y., JI T.R., YAN X.P. Surface Molecular Imprinting on Mn-Doped ZnS Quantum Dots for Room-Temperature Phosphorescence Optosensing of Pentachlorophenol in Water, Analytical Chemistry, 81, 1615, 2009.

26. LONG Q., LI H.T., ZHANG Y.Y., YAO S.Z. Upconversion nanoparticle-based fluorescence resonance energy transfer assay for organophosphorus pesticides, Biosensors \& Bioelectronics, 68, 168, 2015.

27. PLIKAYTIS B.D., TURNER S.H., GHEESLING L.L., CARLONE G.M. Comparisons of Standard Curve-fitting Methods to Quantitate Neisseria-meningitidis Group-A polysaccharide antibody-levels BY enzyme-linkedimmunosorbent-assay, Journal of Clinical Microbiology, 29, 1439, 1991.

28. MIURA K., ORCUTT A.C., MURATOVA O.V., MILLER L.H., SAUL A., LONG C.A., Development and characterization of a standardized ELISA including a reference serum on each plate to detect antibodies induced by experimental malaria vaccines, Vaccine, 26, 193, 2008.

29. PIERMARINI S., MICHELI L., AMMIDA N.H.S., PALLESCHI G., MOSCONE D. Electrochemical immunosensor array using a 96-well screen-printed microplate for aflatoxin B-1 detection, Biosensors \& Bioelectronics, 22, 1434, 2007. 
